\title{
Co-occurrence of frameshift mutations in SMAD6 and TCF12 in a child with complex craniosynostosis
}

\author{
Andrew T. Timberlake (1),2, Robin Wu'2, Carol Nelson-Williams' ${ }^{1}$, Charuta G. Furey' ${ }^{1}$, Kristi I. Hildebrand ${ }^{3}$, Scott W. Elton ${ }^{3}$, \\ Jeyhan S. Wood ${ }^{4}$, John A. Persing ${ }^{2}$ and Richard P. Lifton ${ }^{1,5}$
}

\begin{abstract}
Non-syndromic craniosynostosis (CS) affects 1 in 2350 live births. Recent studies have shown that a significant fraction of cases are caused by de novo or rare transmitted mutations that promote premature osteoblast differentiation in cranial sutures. Rare heterozygous loss-of-function (LOF) mutations in SMAD6 and TCF12 are highly enriched in patients with non-syndromic sagittal and coronal CS, respectively. Interestingly, both mutations show striking incomplete penetrance, suggesting a role for modifying alleles; in the case of SMAD6, a common variant near BMP2 drastically increases penetrance of sagittal CS. Here, we report a proband presenting with both sagittal and coronal craniosynostosis with the highly unusual recurrence of CS within two months of initial surgery, requiring a second operation to re-establish suture patency at six months of age. Exome sequencing revealed a rare transmitted frameshift mutation in SMAD6 (p. 152 fs*27) inherited from an unaffected parent, absence of the common BMP2 risk variant, and a de novo frameshift mutation in TCF12 (p.E548f**14). SMAD6 and TCF12 independently inhibit transcriptional targets of BMP signaling. The findings are consistent with epistasis of these mutations, increasing penetrance and severity of CS in this proband. They also add to the list of composite phenotypes resulting from two Mendelian mutations, and support the utility of exome sequencing in atypical CS cases.
\end{abstract}

The female proband was delivered at term after an uncomplicated pregnancy, and was referred to the pediatric neurosurgical service at birth due to her abnormal head shape. On physical exam, the patient had flattening of the left frontal bone with contralateral frontal bossing and associated harlequin deformity of the left orbit, consistent with left coronal synostosis. In addition, the calvarium posterior to the coronal sutures was elongated and narrow (scaphocephalic), consistent with sagittal synostosis (Fig. 1a, b). A CT scan confirmed

Correspondence: Richard P. Lifton (rickl@rockefeller.edu)

'Department of Genetics, Yale University School of Medicine, New Haven, CT, USA

${ }^{2}$ Section of Plastic and Reconstructive Surgery, Yale University School of Medicine, New Haven, CT, USA

Full list of author information is available at the end of the article. sagittal and left coronal synostosis, and the child underwent endoscopic strip craniectomy at nine weeks of age.

The child was subsequently followed biweekly in neurosurgery clinic and by an orthotist for helmet adjustments to shape skull growth. Two months after surgery, the orthotist noted that the child's left forehead remained flattened and was not rounding as expected. At the child's subsequent neurosurgical clinic visit, the parietal and occipital regions had rounded and expanded as expected; however, the left frontal region had stopped rounding. A repeat head CT was performed, which demonstrated rapid healing and patency of the sagittal suture and refusion of the left coronal suture along with complete fusion of the right coronal suture (Fig. 1c-e). Such rapid recurrence of craniosynostosis is extremely unusual. To correct the anterior deformity, the child underwent a cranial vault reconstruction with fronto-orbital

\section{(c) The Author(s) 2018}

(c) (i) Open Access This article is licensed under a Creative Commons Attribution 4.0 International License, which permits use, sharing, adaptation, distribution and reproduction cc) in any medium or format, as long as you give appropriate credit to the original author(s) and the source, provide a link to the Creative Commons license, and indicate if changes were made. The images or other third party material in this article are included in the article's Creative Commons license, unless indicated otherwise in a credit line to the material. If material is not included in the article's Creative Commons license and your intended use is not permitted by statutory regulation or exceeds the permitted use, you will need to obtain permission directly from the copyright holder. To view a copy of this license, visit http://creativecommons.org/licenses/by/4.0/. 


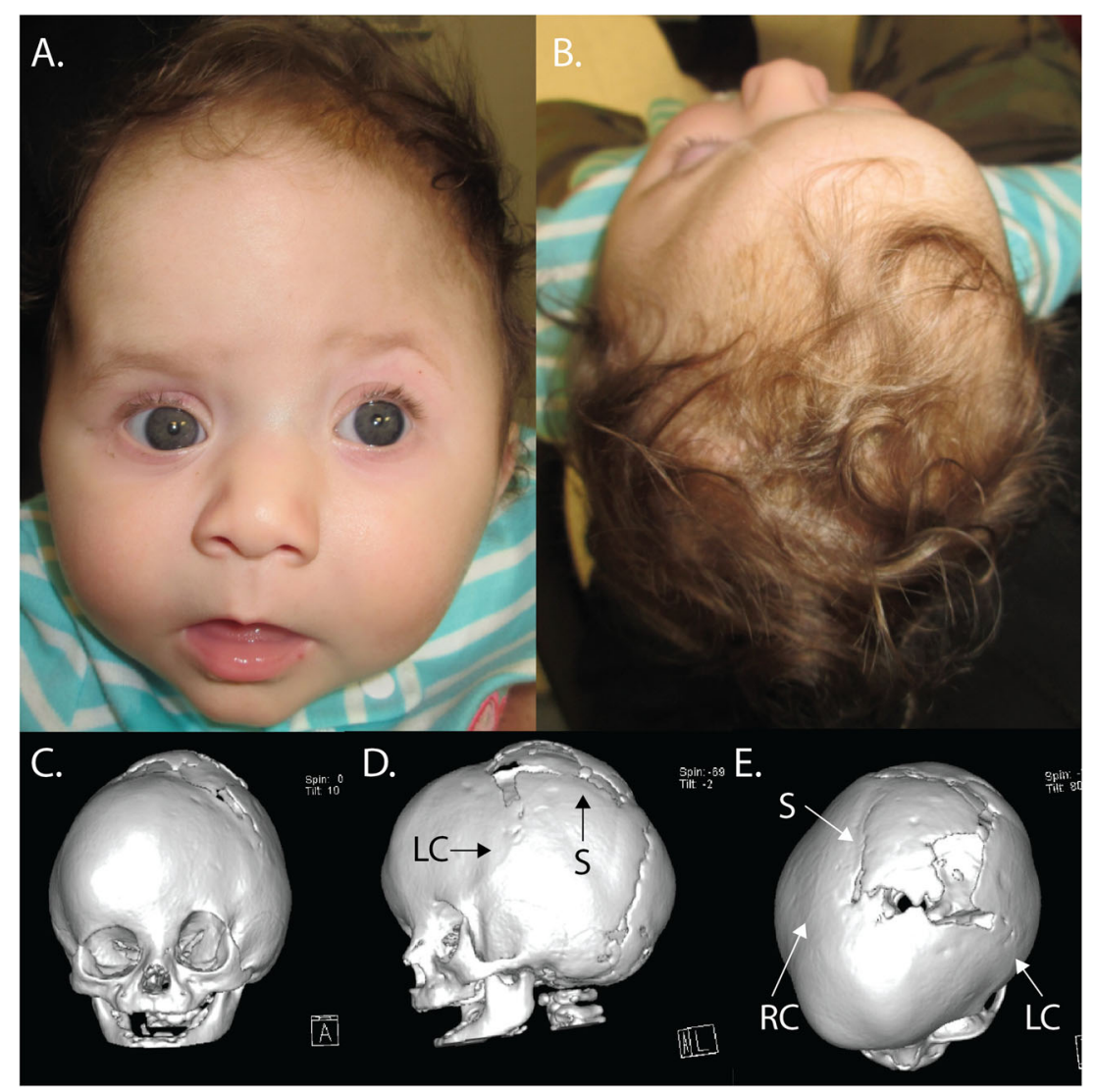

Fig. 1 Patient photographs and 3D CT imaging from a second operation at 6 months of age. a Frontal view demonstrating left coronal synostosis with anterior plagiocephaly (suture fusion causing ipsilateral flattening and contralateral bossing of the forehead). Note the "twisted" appearance of the face as a result of unicoronal synostosis. $\mathbf{b}$ Anterior plagiocephaly as seen from above. Note the bossing of the right forehead and retrusion of the left forehead. Coronal $(\mathbf{c})$, lateral (d), and axial (e) views of the 3D CT reconstruction performed three months after the initial strip craniectomy (age 5.5 months), demonstrating fusion of both the right coronal (RC) and left coronal (LC) sutures and sequelae of the previous strip craniectomy (S)

advancement at six months of age. The child is developing normally to date, and it is unknown at present if she will need further surgical cranial reconstruction.

To explore potential genetic contributions to her condition, we performed whole exome sequencing of the case-parent trio using DNA prepared from buccal swab samples according to standard protocols. Exome capture was performed using the IDT xGen capture reagent, which was followed by 99 base paired-end sequencing on the Illumina HiSeq 2000 instrument. Sequence reads were aligned to the GRCh37/hg19 human reference genome using BWA-Mem. Local realignment and quality score recalibration were performed using the GATK pipeline, after which variants were called using the GATK Haplotype Caller. A Bayesian algorithm, TrioDeNovo, was used to call de novo mutations ${ }^{1}$. VQSR 'PASS' variants with an ExAC allele frequency $\leq 10^{-3}$ sequenced to a depth of eight or greater in the proband and 10 or greater in each parent with Phred-scaled genotype likelihood scores $>30$ and de novo quality scores $\left(\log _{10}\right.$ (Bayes factor) $)>6$ were considered. Independent aligned reads at variant positions were visualized in silico to remove false calls. All retained calls had de novo genotype quality scores of 100 . Transmitted variants were called as per above, and all variants were annotated using $\mathrm{ANNOVAR}^{2}$ with allele frequencies assigned to each variant from the ExAC database ${ }^{3}$.

Analysis showed that the proband had rare heterozygous LOF mutations in both of the two predominant non-syndromic CS genes, SMAD6 and TCF12,5. The mutation in SMAD6 was an early frameshift mutation (p.

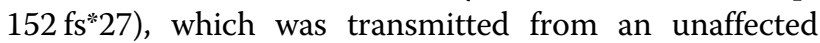
parent (Fig. 2, Table 1). The mutation in TCF12 was also a frameshift mutation (p.K548fs*14), which was de novo. Both mutations were absent from the ExAC and GnomAD databases, which contain $>240,000$ alleles $^{3}$, and both mutations were confirmed by Sanger sequencing (Fig. 2). No other compelling heterozygous rare LOF or damaging missense variants were identified, and no rare recessive genotypes were identified (Table 1, Supplementary Table 1 ). 
A.

\section{$\square$ CRANIOSYNOSTOSIS}

SMAD6: c.455_461del:p.P152fs*27 TCF12: c.1639_1642del:p.E548fs*14

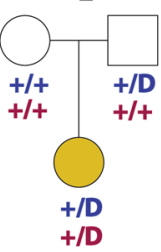

c.

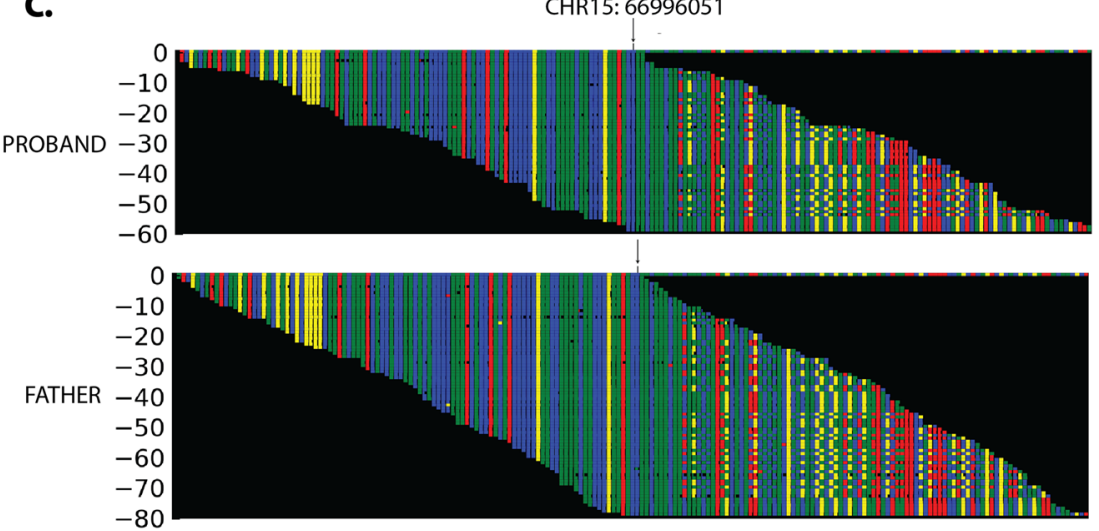

Fig. 2 Heterozygous LOF mutations in SMAD6 and TCF12 in a proband with complex craniosynostosis. a Pedigree and genotypes. Genotypes of each subject are shown: SMAD6 genotypes are in blue, and TCF12 genotypes are in red. " +" and "D" denote the wild-type and indicated frameshift alleles, respectively. No member of the trio harbored the BMP2 risk SNP ' $C^{\prime}$ at rs 1884302 . The SMAD6 p.152 fs*27 mutation was transmitted from an unaffected parent, and the TCF12 p.E548fs*14 mutation arose de novo in the proband. b Confirmation of the de novo TCF12 mutation. Sanger sequencing traces of PCR amplicons containing the TCF12 mutation identified by exome sequencing are shown. The mutation identified in the DNA sequence and its impact on TCF12 protein (in single letter code) are indicated above the trace. The deleted bases are denoted, and they result in an overlap of wild-type and mutant sequences. Both the mother and father's traces demonstrate the wild-type TCF12 sequence, whereas the proband has a de novo 4-bp deletion that results in a frameshift. c In silico visualization of the SMAD6 frameshift deletion in the proband. Sequence reads derived from single molecules on the Illumina platform are shown. The reference sequence of a segment of SMAD6 that includes base 15:66996051 (denoted by arrow) is shown in the top row, and red, blue, green and yellow squares represent the bases A, C, G, and T, respectively. Below, all independent reads that map to this interval are shown. The results show that the proband and father both have a 7-bp deletion that causes a frameshift in the SMAD6 coding sequence

Table 1 Rare loss-of-function variants identified in a child with complex craniosynostosis

\begin{tabular}{|c|c|c|c|c|c|c|c|c|}
\hline Gene name & Chrom & Position & Ref & Alt & Mutation class & Impact & ExAC frequency & pLI \\
\hline RIIAD1 & 1 & 151694016 & G & $\mathrm{T}$ & stopgain & p. E2X & Novel & NA \\
\hline ENPP6 & 4 & 185033931 & AT & - & frameshift deletion & p. M296fs & Novel & 0 \\
\hline SMOC1 & 14 & 70418934 & GCAGGTCCTAC & - & frameshift deletion & p. G60fs & Novel & 0.01 \\
\hline TCF12 & 15 & 57555366 & AAAG & - & frameshift deletion & p. E548fs & Novel & 0.97 \\
\hline SMAD6 & 15 & 66996051 & CGGCGGG & - & frameshift deletion & p. P152fs & Novel & 0 \\
\hline ZNF551 & 19 & 58199463 & G & - & frameshift deletion & p. R579fs & Novel & 0 \\
\hline
\end{tabular}

Table containing all rare (ExAC frequency $<2 \times 10^{-5}$ ) LOF variants identified in a child with complex craniosynostosis. Two novel LOF variants in previously identified craniosynostosis genes (SMAD6 and TCF12) were identified. The novel TCF12 frameshift mutation was discovered to have arisen de novo in the proband (Fig. 2). 


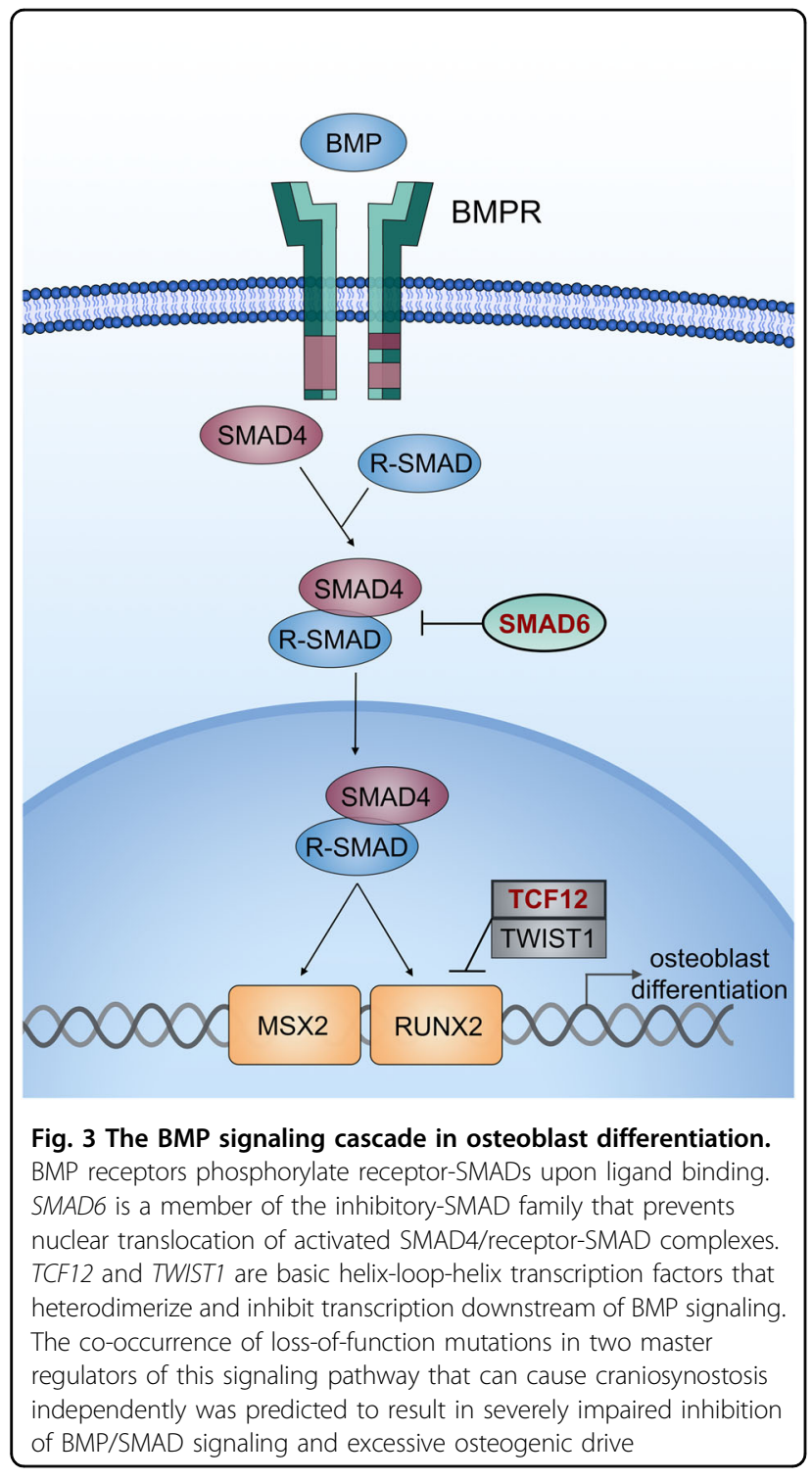

Heterozygous TCF12 mutations have been previously shown to cause coronal CS with considerable phenotypic overlap with Saethre-Chotzen syndrome ${ }^{4}$, which is caused by LOF mutation in TWIST1, which heterodimerizes with TCF12 to inhibit transcription downstream of BMP signaling. Similar LOF mutations in TCF12 were subsequently identified in patients with nonsyndromic coronal craniosynostosis ${ }^{6}$. De novo or transmitted LOF mutations in SMAD6 are found in $\sim 6 \%$ of non-syndromic midline craniosynostosis cases $^{5}$. LOF mutations in both TCF12 and SMAD6 both show striking incomplete penetrance $(\sim 40$ and $20 \%$ penetrance, respectively $)^{4,7}$. In the case of $S M A D 6$, epistatic interaction with a common risk variant near BMP2, which by itself has modest effect on risk, increases penetrance to $>90 \%{ }^{5,7}$. The BMP2 rs 1884302 locus was genotyped in the proband and both parents, and no family members harbored the CS risk allele ' $C$ ', consistent with the parent harboring the SMAD6 mutation being free of CS (Fig. 2).

The combination of rare LOF mutations at established Mendelian loci (SMAD6 and TCF12) in the proband was particularly interesting ${ }^{8}$. While SMAD6 has long been known as an inhibitory-SMAD that negatively regulates BMP signaling, TCF12 silencing in mesenchymal stem cells was only recently shown to result in increased phosphorylation of receptor-SMADs, implying that loss of TCF12 function also augments BMP signaling via the BMP/SMAD axis ${ }^{9}$. This finding suggests that the combination of SMAD6 and TCF12 haploinsufficiency increases BMP signaling to levels substantially greater than those seen with either mutation alone, sufficient to ensure penetrance at both the sagittal and coronal sutures (Fig. 3). Moreover, while neither SMAD6 nor TCF12 haploinsufficiency in isolation has been associated with increased rates of reoperation ${ }^{4,5}$, we propose that these mutations together promote sufficiently high osteogenic drive to promote the very unusual rapidity of recurrent synostosis after surgery (Figs. 1, 3).

The combination of a common $B M P 2$ variant with $L O F$ variants in SMAD6 is sufficient to push BMP/SMAD signaling to levels sufficient to cause suture fusion ${ }^{5}$. The present results suggest that alleles other than the common $B M P 2$ risk variant can have epistasis with rare SMAD6 alleles. It seems compelling that the combination of a SMAD6 LOF mutation with loss of an independent inhibitor of BMP signaling via TCF12 mutation produces particularly high BMP/SMAD signaling and a strikingly more severe phenotype than SMAD6 LOF mutation alone (Figs. 1, 3). It will be interesting to see whether other patients with non-syndromic complex CS also have mutations in these two genes.

While several factors, such as patient age at presentation, suture fusion pattern, and patient co-morbidities, play a role in which type of surgery (endoscopic versus open) is offered to craniosynostosis patients, knowing the genetic results for specific patients could prove useful in guiding operative planning for this complex patient population. The goals of cranial vault reconstruction are to obtain an aesthetically pleasing shape of the skull that will allow adequate growth of the brain in ideally one operation. Identification of high risk genotypes prior to surgery-particularly in cases with unusual clinical features-may prove useful in guiding surgical management in the future and may enable more informed discussions with patients' families.

\section{HGV Database}

The relevant data from this Data Report are hosted at the Human Genome Variation Database at https://doi.org/10.6084/m9.figshare.hgv.2330 https://doi. org/10.6084/m9.figshare.hgv.2333. 


\section{Acknowledgements}

The study protocol was approved by the Yale Human Investigation Committee Institutional Review Board, and consent for use of patient photographs was obtained by the treating physicians. This project was supported by the Yale Center for Mendelian Genomics (NIH Grant M\#UM1HG006504-05), the NIH Medical Scientist Training Program (NIH/National Institute of General Medical Sciences Grant T32GM007205), and the Howard Hughes Medical Institute.

\section{Author details}

'Department of Genetics, Yale University School of Medicine, New Haven, CT, USA. ${ }^{2}$ Section of Plastic and Reconstructive Surgery, Yale University School of Medicine, New Haven, CT, USA. ${ }^{3}$ Division of Pediatric Neurosurgery, University of North Carolina School of Medicine, Chapel Hill, NC, USA. ${ }^{4}$ Division of Plastic and Reconstructive Surgery, University of North Carolina School of Medicine Chapel Hill, NC, USA. 'Laboratory of Human Genetics and Genomics, The Rockefeller University, New York, NY, USA

\section{Conflict of interest}

The authors declare that they have no conflict of interest.

\section{Publisher's note}

Springer Nature remains neutral with regard to jurisdictional claims in published maps and institutional affiliations.

Supplementary information is available for this paper at https://doi.org/ 10.1038/s41439-018-0014-X.
Received: 25 April 2018 Revised: 29 May 2018 Accepted: 2 June 2018. Published online: 28 June 2018

\section{References}

1. Wei, Q. et al. A Bayesian framework for de novo mutation calling in parentsoffspring trios. Bioinformatics 31, 1375-1381 (2015).

2. Wang, K., Li, M. \& Hakonarson, H. ANNOVAR: functional annotation of genetic variants from high-throughput sequencing data. Nucleic Acids Res. 38, e164 (2010).

3. Lek, M. et al. Analysis of protein-coding genetic variation in 60,706 humans Nature 536, 285-291 (2016).

4. Sharma, V. P. et al. Mutations in TCF12, encoding a basic helix-loop-helix partne of TWIST1, are a frequent cause of coronal craniosynostosis. Nat. Genet. 45, 304-307 (2013).

5. Timberlake, A. T. et al. Two locus inheritance of non-syndromic midline craniosynostosis via rare SMAD6 and common BMP2 alleles. ELife 5, e20125 (2016).

6. Wilkie, A. O. M., Johnson, D. \& Wall, S. A. Clinical genetics of craniosynostosis. Curr. Opin. Pediatr. 29, 622-628 (2017)

7. Timberlake, A. T. et al. De novo mutations in inhibitors of Wnt, BMP, and Ras/ ERK signaling pathways in non-syndromic midline craniosynostosis. Proc. Nat Acad. Sci. USA 114, E7341-E7347 (2017).

8. Posey, J. E. et al. Resolution of disease phenotypes resulting from multilocus genomic variation. N. Engl. J. Med. 376, 21-31 (2017).

9. Yi, S., Yu, M., Yang, S., Miron, R. J. \& Zhang, Y. Tcf12, a member of basic helixloop-helix transcription factors, mediates bone marrow mesenchymal stem cell osteogenic differentiation in vitro and in vivo. Stem Cells 35, 386-397 (2017). 